\title{
Quantum Repeater Node Demonstrating Unconditionally Secure Key Distribution
}

\author{
S. Langenfeld $\odot,{ }^{*}$ P. Thomas, O. Morin $\odot$, and G. Rempe $\odot$ \\ Max-Planck-Institut für Quantenoptik, Hans-Kopfermann-Strasse 1, 85748 Garching, Germany
}

(Received 23 December 2020; accepted 13 May 2021; published 11 June 2021)

\begin{abstract}
Long-distance quantum communication requires quantum repeaters to overcome photon loss in optical fibers. Here we demonstrate a repeater node with two memory atoms in an optical cavity. Both atoms are individually and repeatedly entangled with photons that are distributed until each communication partner has independently received one of them. An atomic Bell-state measurement followed by classical communication serves to establish a key. We demonstrate scaling advantage of the key rate, increase the effective attenuation length by a factor of 2 , and beat the error-rate threshold of $11 \%$ for unconditionally secure communication, the corner stones for repeater-based quantum networks.
\end{abstract}

DOI: 10.1103/PhysRevLett.126.230506

Optical repeaters based on light amplification have been a game changer for the development of modern telecommunication links, enabling fiber-based networks at the global scale. The same is to be expected for their quantum-physical counterparts, namely quantum repeaters. In both cases, the issue is to overcome the propagation loss in long-distance transmission lines. As quantum signals, the qubits, cannot be amplified or copied [1], the classical repeater strategy fails for quantum links. The challenge was resolved by Briegel et al. [2] who proposed a quantum repeater protocol that can distribute entanglement, the basic resource for quantum networks, between quantum memories $[3,4]$.

The main idea behind a repeater [2,5] is to replace the probabilistic transmission through the quantum channel by a heralded preparation of the quantum link followed by deterministic classical communication. Towards this goal, the link is divided into distinct segments connected by repeater nodes. These nodes serve to independently prepare each segment in an entangled state that can be used for communication. The subsequent concatenation of all segments by entanglement swapping then improves the rateversus-distance scaling for the channel transmission in a fundamental way. Of course, this repeater advantage is in vain if the efficiency is small and the errors are large. Hence, high-fidelity operations are essential for quantum repeaters. Moreover, as the preparation of the segments is achieved probabilistically with a heralded repeat-untilsuccess strategy, synchronization of all segments requires repeater nodes with long-lived qubit memories.

Published by the American Physical Society under the terms of the Creative Commons Attribution 4.0 International license. Further distribution of this work must maintain attribution to the author(s) and the published article's title, journal citation, and DOI. Open access publication funded by the Max Planck Society.
The necessary elements for a scalable quantum repeater such as light-matter entanglement [6], qubit memories [7], Bell-state measurements (BSMs) [8], entanglement swapping [9], and distillation [10] have been investigated individually for different platforms and a plethora of protocols [11-13]. However, up to date, there has been no experimental demonstration of the combination of these ingredients into a single repeater protocol, mostly due to technical limitations concerning efficiency and fidelity, incompatibility of different qubit carriers, or irreconcilability of the individual steps of the protocol.

Reaching the goal of a repeater-increased communication distance remains a grand challenge that needs to be addressed step by step. Quantum key distribution (QKD) provides an ideal and application-friendly setting for this approach [14], with the practical advantage that end nodes are implemented as classical parties, Alice and Bob, instead of quantum memories. In QKD, two parties establish a secret key that is unconditionally secure against attacks by adversary eavesdroppers provided the quantum bit error rate (QBER), i.e., the infidelity of the quantum link, remains below the threshold of $11 \%$ [15]. A useful corollary is that distillation can be performed classically on the obtained key. Towards surpassing the rate-versusdistance scaling of direct transmission, specific protocols were proposed [16-18], and various platforms were analyzed [19], but experimental work is still elusive: One demonstration showed an improved scaling but is fundamentally limited to a single node [20], another employs a memory but the required QBER was not achieved and the scalability to multiple nodes remains uncertain [21].

Here we combine state-of-the-art quantum-optical techniques to experimentally approach the protocol proposed by Luong et al. [17]. This protocol examines a modular building block that can be concatenated to construct a quantum repeater which, considering only channel losses, is scalable to larger distances $[19,22,23]$. We realize the 
core element, dubbed a quantum repeater node, with two segments that connect to Alice and Bob. It achieves the necessary QBER to distribute unconditionally secure keys with a probability that scales with distance more favorable, i.e., proportional to the square root of the direct transmission probability. The implementation relies heavily on the toolbox provided by cavity quantum electrodynamics (QED) [24]. Most importantly, the optical cavity acts as a light-matter quantum interface for the efficient generation of atom-photon entanglement [25], and two individually and repeatedly addressable intracavity atoms serve as two distinct high-fidelity qubit memories [26]. The BSM again relies on cavity QED and the common cavity mode in which the atoms are localized. A further advantage for future experiments is the demonstrated possibility to perform quantum-logic gates [27], e.g., for entanglement purification.

As depicted in Fig. 1, the experimental sequence is divided into four parts [17]: First, atom $A$ is initialized and subsequently transferred into an atom-photon

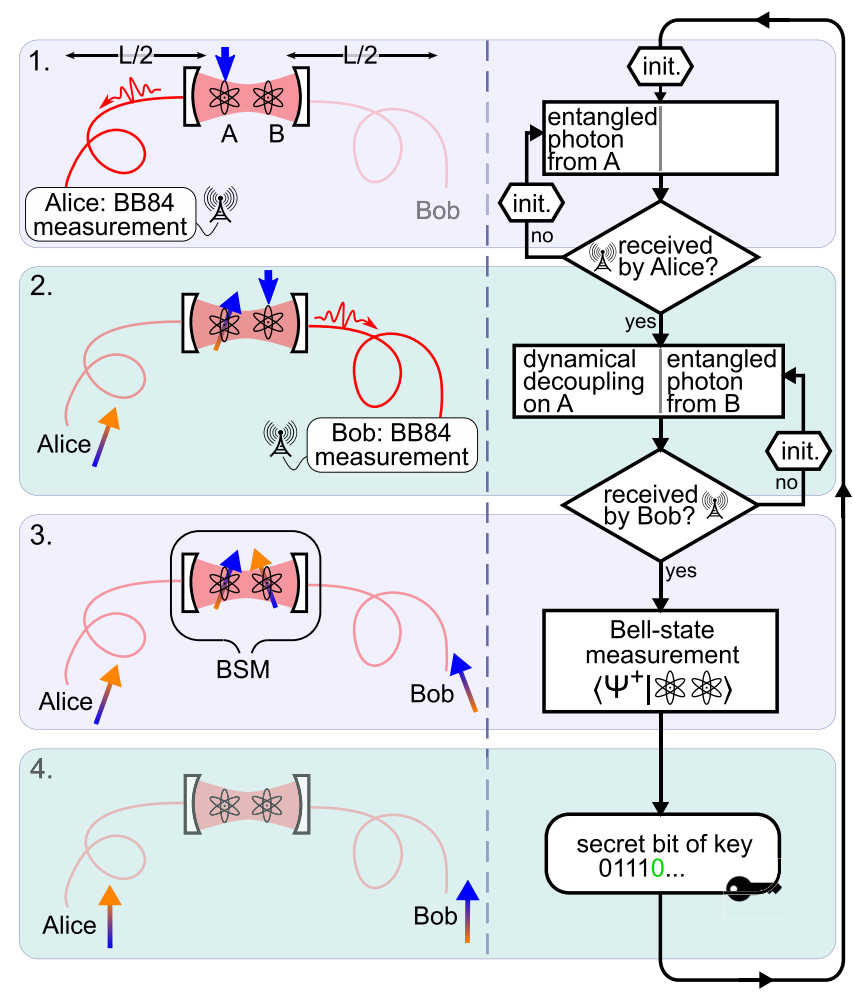

FIG. 1. Quantum-repeater scheme for distributing a string of secret bits. Two atoms in a cavity serve as a repeater node. Atom $A$ repeatedly creates atom-photon entanglement and sends the photon to Alice until she announces (antenna) the detection of a photon in a BB84 measurement (1), thus creating a correlation between atom $A$ and Alice (indicated by a color-correlated spin pair of same angle). The same for atom $B$ and Bob (2) while the qubit stored in atom $A$ is protected by dynamical decoupling. A local Bell-state measurement (BSM) on the two atoms swaps the two correlations to Alice and Bob (3), who share a secret key after classical postprocessing (4). polarization-entangled pair. The photon travels via an optical fiber from the repeater node to one of the communication partners, Alice, where it is detected in a polarization-resolving measurement setup. Following the BB84 protocol [28], Alice randomly chooses between two nonorthogonal detection bases, thereby establishing a correlation between the state of atom $A$ and the measurement result of Alice. In Fig. 1 this is highlighted via color-correlated spins of the same angle. If Alice did not detect a photon, e.g., due to fiber transmission losses, this failure is communicated back to the repeater node in order to restart the sequence with initialization and entanglement generation.

Conversely, a photon detected by Alice heralds a successful transmission and signals the repeater node to continue with step two. This consists of the same sequence, but now applied to atom $B$ and the other communication partner, Bob. While atom $B$ repeatedly tries to connect to Bob, the previously established correlation between Alice and atom $A$ needs to be preserved. The maximum number of entanglement attempts on atom $B, n$, is therefore limited by the coherence time of the memory atom $A$ and potential cross talk between the two atoms. This number can be increased by extending the qubit coherence time, e.g., by applying dynamical decoupling on atom $A$ while trying to connect atom $B$ with Bob. If within $n$ trials no photon was detected, the whole sequence is aborted and restarts with the initialization of atom $A$.

After successful detection of two photons, one by Alice and one by Bob, the repeater node carries one qubit in each memory, one correlated with Alice and the other with Bob. In this case the third step of the sequence proceeds with the $\mathrm{BSM}$ of the two atoms. This swaps the correlation from atom $A$-Alice and atom $B$-Bob to Alice-Bob, leaving no trace of the correlation in the repeater node. Finally, in the forth and last step the result of the BSM is publicly announced to Alice and Bob which then share one more bit of raw, i.e., not yet secured, key. A secret key can be obtained after classical error correction and privacy amplification [29].

We emphasize that the described protocol can straightforwardly be extended to distribute entanglement between Alice and Bob by replacing the BB84 photon-absorbing end nodes with heralded qubit memories [30,31]. Most importantly for quantum networks, the protocol is scalable to a chain of repeater nodes connecting Alice and Bob, e.g., by simply interfering photons from neighboring nodes on a beam splitter [23]. This would further amplify the scaling advantage.

The experiment starts by loading two ${ }^{87} \mathrm{Rb}$ atoms close to the center of the optical cavity where they are trapped in a two-dimensional optical lattice [32]. Both atoms couple about equally to the cavity mode, while classical light fields can be applied globally or individually via an optical addressing system [Fig. 2(a)]. In our proof-of-principle experimental demonstration, a single detection setup plays the role of both Alice and Bob. A fast electro-optical 
(a)

(b)
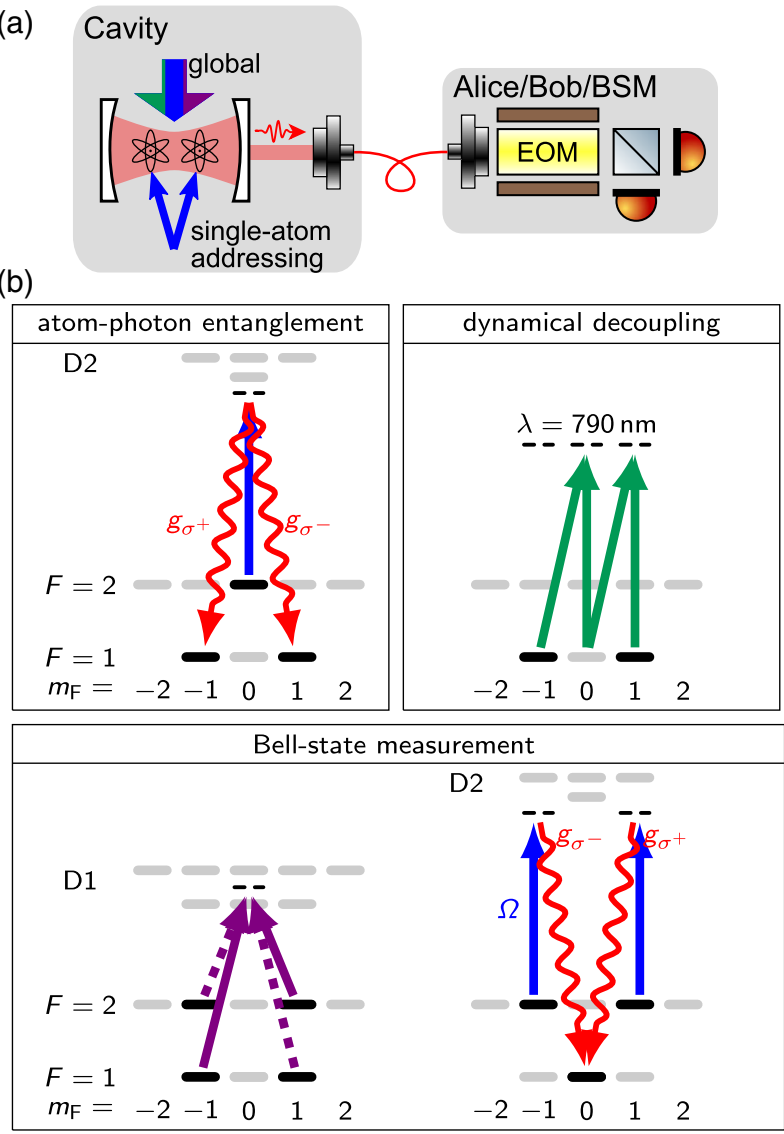

FIG. 2. Implementation of and toolbox for a quantum-repeater protocol. (a) Sketch of the experimental setup. Two ${ }^{87} \mathrm{Rb}$ atoms in a high-finesse cavity serve as matter qubits and can either be addressed individually or globally. A single detection setup is used for Alice, Bob, and the photonic BSM with an electrooptical modulator (EOM) for fast polarization basis selection. (b) Level diagrams and relevant optical fields for the steps described in Fig. 1.

modulator (EOM) is used to switch the polarization analysis basis between two of the Pauli eigenbases, i.e., $X$ and $Z$. The same detection setup is later on used for the measurement of Bell states in the $Z$ basis.

The sequence begins by initializing the atom in the ground state $\left|F=2, m_{F}=0\right\rangle$, from which it generates an atom-photon spin-polarization-entangled state via a vacuum-stimulated Raman adiabatic passage (VSTIRAP) [25] to $\left|F=1, m_{F}= \pm 1\right\rangle$ [Fig. 2(b)]. In order to avoid unintended cross talk between the two atoms, we employ a large single-photon detuning of $-200 \mathrm{MHz}$ with respect to the excited atomic state $\left|5^{2} P_{3 / 2}, F^{\prime}=1, m_{F}= \pm 1\right\rangle$. Moreover, applying the control laser pulse selectively to only the wanted atom avoids cross-illumination between the atoms [26]. Because of constraints in the atom cooling and trapping [32], entanglement generation is repeated a maximum of $n$ times.

In the second step of the protocol (Fig. 1), atom $B$ has to be repeatedly reinitialized without affecting the qubit already stored in atom $A$. This is achieved by atomselective pumping from $|F=1\rangle$ to $|F=2\rangle$ via the optical addressing system. At the same time, Zeeman pumping to $\left|m_{\mathrm{F}}=0\right\rangle$ is applied globally, i.e., without being atom selective, as the employed transition $|F=2\rangle \leftrightarrow$ $\left|5^{2} P_{1 / 2}, F^{\prime}=2\right\rangle$ is $6.8 \mathrm{GHz}$ detuned from the qubitcarrying states in $|F=1\rangle$. The initialization is optimized to be fast, to have as little cross talk as possible, but still maintain a high efficiency. We achieved a single-trial success probability for zero communication distance, $L=0$, of $p_{A B, L=0}=(22.13 \pm 0.03) \%$. This approximately matches the combination of the individually obtained values for the atom initialization (66\%), photon generation (69\%), fiber coupling including optical elements (85\%), and detection efficiency (68\%) [32]. The whole atom initialization takes $8 \mu \mathrm{s}$, followed by $2 \mu \mathrm{s}$ for photon emission and an additional waiting time of up to $10 \mu \mathrm{s}$ for receiving the heralding signal from Alice or Bob. Thus, the atom-photon entanglement attempts are repeated every $20 \mu \mathrm{s}$.

Qubit coherence time is a very important aspect for any memory-based quantum-repeater architectures [36]. Here, we use a dynamical decoupling scheme [Fig. 2(b)] to improve the coherence time from below $1 \mathrm{~ms}$ [26] to above $20 \mathrm{~ms}$. This extension is both necessary and sufficient for the protocol implemented here. Details are described in [32].

After successfully creating correlations in both segments, i.e., Alice with atom $A$ and Bob with atom $B$, a BSM swaps the correlation to Alice and Bob. We perform a linear-optics BSM [8] on photons carrying the qubit information of atoms $A$ and $B$ while the common cavity mode erases the which-way (which-atom) information from the photons. More specifically, in order to drive a VSTIRAP for qubit-readout starting from $|F=2\rangle$, we first map both atomic qubits simultaneously from $\mid F=1$, $\left.m_{F}= \pm 1\right\rangle$ to $\left|F=2, m_{F}=\mp 1\right\rangle$ via a two-photon Raman transition [Fig. 2(b)]. Afterwards, the VSTIRAP with the global control beam generates two photons which are ideally indistinguishable as they originate from atoms in the same cavity mode and are driven by the same control beam. The detection of one photon in state $\left|\uparrow_{z}\right\rangle$ and the other photon in state $\left|\downarrow_{Z}\right\rangle$ heralds the symmetric Bell state $\left|\Psi^{+}\right\rangle=\left(\left|\uparrow_{z} \downarrow_{z}\right\rangle+\left|\downarrow_{z} \uparrow_{z}\right\rangle\right) / \sqrt{2}[32]$ and thus selects those cases where Alice and Bob must have obtained (anti-) correlated results if both had measured in the $(Z) X$ basis. This BSM, which involves the generation and detection of two photons, has an efficiency of $p_{\mathrm{BSM}}=(5.07 \pm 0.03) \%$, including the $50 \%$ detection limitation of linear optics.

Experimental results are depicted in Fig. 3. First, we analyze the yield of the key generation process, i.e., how many raw bits are generated per channel use, and also compare it to the achievable rate when using direct transmission with a setup using the same efficiencies for photon generation and detection. In contrast to Ref. [21], we do not attribute the system inefficiencies to an effective distance. 
Instead, we consider as equivalent distance only the losses we add on top of our experimental imperfections. This results in a curve starting at $L=0$. Figure 3(a) shows the achieved yield for different cutoffs in the number of trials $N \leq n$. Following the usual convention [17], we evaluate the number of trials as $N=\max \left(N_{A}, N_{B}\right)$, i.e., the maximum number of trials needed by atom $A$ and atom $B$. For small $n$, i.e., without fully utilizing the repeat-until-success strategy, the rate decays very similarly to direct transmission, but with an offset that is given by the unavoidable inefficiency. For $n=40 \gg 1 / p_{A B, L}$, i.e., effectively without cutoff in the examined range of $L$, the experimental data follow the theoretical expectation with a raw key rate that

(a)
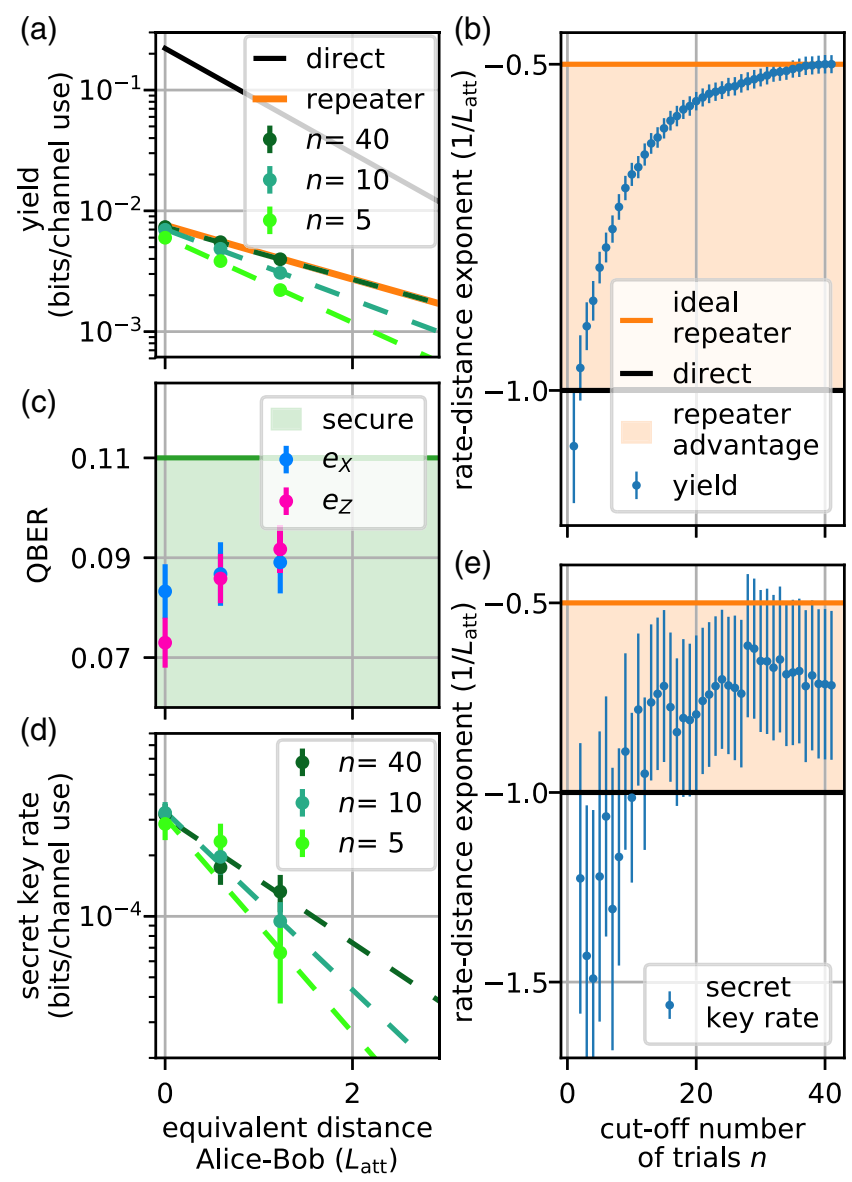

FIG. 3. Creating an unconditionally secure key with repeater advantage. (a) Yield of the repeater protocol for different distances and three exemplary cutoffs in the number of trials, $n$. Dashed lines show fits to the data. Theory curves for the repeater case and the direct transmission case serve as comparison. (b) Slope of the fits given in (a), quantifying the yield-versusdistance scaling as a function of the maximum number of trials, $n$. With increasing $n$, the exponent changes from $-1 / L_{\text {att }}$ (direct transmission) to $-0.5 / L_{\text {att }}$ (repeater advantage). (c) For both detection bases the quantum bit error rate (QBER) beats the threshold of $11 \%$ for unconditional security. (d),(e) Same as (a), (b) for secret key rate. All error bars represent 1 standard deviation of the statistical uncertainty (details in [32]). follows a scaling $\exp \left[-L /\left(2 L_{\text {att }}\right)\right]$ instead of $\exp \left(-L / L_{\text {att }}\right)$ for direct transmission. Here, $L_{\text {att }}$ is the attenuation length of the optical fiber. The scaling advantage is further highlighted in Fig. 3(b). By increasing the maximum number of trials the data demonstrate a smooth transition from the direct-transmission regime to the memory-assisted regime. Here, the absolute raw key rate is 0.57 bits/s [32].

In order to establish an unconditionally secure key between Alice and Bob, the experiment aims at a QBER below $11 \%[14,15]$. We analyze this by comparing the obtained (anti-) correlation of Alice and Bob with the theoretically expected one. The error rates $e$ are given in Fig. 3(c) for the two chosen BB84 detection bases $X$ and $Z$ which, following the earlier introduced atom-photon entanglement protocol [Fig. 2(b)], correspond to atomic superposition and energy eigenstates, respectively. For all distances, we beat the $11 \%$ threshold by at least 3 standard deviations. In order to achieve this, we observe that in our BSM the error rate increases with the time separation between the two photon-detection events. Thus only trials with a sufficiently small detection-time difference are used for secret-key generation [32]. This reduces the secret-key rate by about a factor of 4 . From single-atom single-photon characterization experiments, we estimate that about $8 \%$ of the total QBER is due to infidelities in the atom-photon entanglement generation mechanism governed by our atom-cavity parameters and off-resonant scattering in the employed Raman sequences. Thus, the polarization alignment error and the reduction in visibility of the BSM are negligible in comparison $(\lesssim 1 \%)$ using the selection on small detection-time differences explained above.

More fundamentally, the secret key rate is given by the product of yield and secret-key fraction, $r_{S}$, which is lower bounded by [15]

$$
r_{S}=\frac{1}{2}\left[1-h\left(e_{X}\right)-h\left(e_{Z}\right)\right],
$$

where $h$ is the binary Shannon entropy. The factor $1 / 2$ accounts for the use of two modes (in our case polarizations) per transmitted qubit. Note that this formula assumes perfect classical error correction and infinite length keys. Supporting the threshold introduced above, $r_{S}$ drops to 0 at $e_{X}=e_{Z}=11 \%$. The resulting secret key rate is given in Fig. 3(d), again for different cutoffs in the number of trials. While the overall rate is reduced by about 2 orders of magnitude due to the finite secret-key fraction and the factor 4 for the fraction of usable BSMs explained above, the scaling advantage still unfolds for increasing $n$.

This is further quantified in Fig. 3(e) where, similarly to the yield, the rate-versus-distance exponent is plotted for different cutoffs $n$. For small $n$, our protocol performs worse than direct transmission for which we assume no imperfections. This is due to the finite QBER which increases with distance and thus reduces the secret-key 
fraction for larger distances. However, with increasing $n$ the advantage given by the repeat-until-success strategy unfolds. Although the exponent does not reach the ideal limit as nicely as for the yield, our experiment beats the fundamental limitation regarding scalability of direct transmission. Further analysis of the results of Fig. 3 can be found in [32].

In summary, we have realized a quantum repeater node for unconditionally secure quantum key distribution and have observed a twofold improvement of the rate-versusdistance scaling. As an outlook, we address the question how much improvement is needed to beat direct transmission in absolute rate. Using the model described in Ref. [17] we estimate that this is possible by doubling the memory time to $40 \mathrm{~ms}$, the BSM efficiency to $10 \%$, and increasing the protocol fidelity by $2 \%$ [32]. For these parameters a repeater advantage unfolds for a communication length larger than $L \sim 7 L_{\text {att }}$. At this distance the secret key rate then amounts to $5 \times 10^{-5}$ bits per channel use. The required improvements of the system performance seem feasible, especially for the memory time where values exceeding $100 \mathrm{~ms}$ have been achieved [33]. However, the average number of repeated trials has to be increased to about 150 , a presently intolerably high value for which the atom would quickly be heated out of the trap. Hence, future experiments require better atom trapping and cooling, e.g., with optical tweezers [37]. Once these improvements are implemented, we can investigate the scaling to a chain of repeater nodes $[22,23]$. Another perspective is to increase the number of atoms to more than two to boost the transmission rate [38] or possibly link them to more than two communication partners, e.g., for achieving a quantum conference key agreement [39]. In combination with quantum-logic gates [27] for entanglement purification and heralded quantum memories [31] as end nodes, the door towards full quantum repeaters seems open.

This work was supported by the Bundesministerium für Bildung und Forschung via the Verbund Q.Link.X (16KIS0870), by the Deutsche Forschungsgemeinschaft under Germany's Excellence Strategy-EXC-2111390814868, and by the European Union's Horizon 2020 research and innovation programme via the project Quantum Internet Alliance (QIA, GA No. 820445).

*Corresponding author. stefan.langenfeld@mpq.mpg.de

[1] W. K. Wootters and W. H. Zurek, A single quantum cannot be cloned, Nature (London) 299, 802 (1982).

[2] H.-J. Briegel, W. Dür, J. I. Cirac, and P. Zoller, Quantum Repeaters: The Role of Imperfect Local Operations in Quantum Communication, Phys. Rev. Lett. 81, 5932 (1998).

[3] H. J. Kimble, The quantum internet, Nature (London) 453, 1023 (2008).
[4] S. Wehner, D. Elkouss, and R. Hanson, Quantum internet: A vision for the road ahead, Science 362, eaam9288 (2018).

[5] L.-M. Duan, M. D. Lukin, J. I. Cirac, and P. Zoller, Longdistance quantum communication with atomic ensembles and linear optics, Nature (London) 414, 413 (2001).

[6] L.-M. Duan and C. Monroe, Quantum networks with trapped ions, Rev. Mod. Phys. 82, 1209 (2010).

[7] K. Heshami, D. G. England, P. C. Humphreys, P. J. Bustard, V. M. Acosta, J. Nunn, and B. J. Sussman, Quantum memories: Emerging applications and recent advances, J. Mod. Opt. 63, 2005 (2016).

[8] S. Pirandola, J. Eisert, C. Weedbrook, A. Furusawa, and S. L. Braunstein, Advances in quantum teleportation, Nat. Photonics 9, 641 (2015).

[9] J.-W. Pan, D. Bouwmeester, H. Weinfurter, and A. Zeilinger, Experimental Entanglement Swapping: Entangling Photons That Never Interacted, Phys. Rev. Lett. 80, 3891 (1998).

[10] N. Kalb, A. A. Reiserer, P. C. Humphreys, J. J. W. Bakermans, S. J. Kamerling, N. H. Nickerson, S. C. Benjamin, D. J. Twitchen, M. Markham, and R. Hanson, Entanglement distillation between solid-state quantum network nodes, Science 356, 928 (2017)

[11] Z.-S. Yuan, Y.-A. Chen, B. Zhao, S. Chen, J. Schmiedmayer, and J.-W. Pan, Experimental demonstration of a BDCZ quantum repeater node, Nature (London) 454, 1098 (2008).

[12] N. Sangouard, C. Simon, H. de Riedmatten, and N. Gisin, Quantum repeaters based on atomic ensembles and linear optics, Rev. Mod. Phys. 83, 33 (2011).

[13] Y.-F. Pu, S. Zhang, Y.-K. Wei, N. Jiang, W. Chang, C. Li, and L.-M. Duan Experimental demonstration of memoryenhanced scaling for entanglement connection of quantum repeater segments, Nat. Photonics 15, 374 (2021).

[14] F. Xu, X. Ma, Q. Zhang, H.-K. Lo, and J.-W. Pan, Secure quantum key distribution with realistic devices, Rev. Mod. Phys. 92, 025002 (2020).

[15] P. W. Shor and J. Preskill, Simple Proof of Security of the BB84 Quantum Key Distribution Protocol, Phys. Rev. Lett. 85, 441 (2000).

[16] C. Panayi, M. Razavi, X. Ma, and N. Lütkenhaus, Memoryassisted measurement-device-independent quantum key distribution, New J. Phys. 16, 043005 (2014).

[17] D. Luong, L. Jiang, J. Kim, and N. Lütkenhaus, Overcoming lossy channel bounds using a single quantum repeater node, Appl. Phys. B 122, 96 (2016).

[18] M. Lucamarini, Z. L. Yuan, J. F. Dynes, and A. J. Shields, Overcoming the rate-distance limit of quantum key distribution without quantum repeaters, Nature (London) 557, 400 (2018).

[19] P. van Loock, W. Alt, C. Becher, O. Benson, H. Boche, C. Deppe, J. Eschner, S. Höfling, D. Meschede, P. Michler, F. Schmidt, and H. Weinfurter, Extending quantum links: Modules for fiber- and memory-based quantum repeaters, Adv. Quantum Technol. 3, 1900141 (2020).

[20] M. Minder, M. Pittaluga, G. L. Roberts, M. Lucamarini, J. F. Dynes, Z. L. Yuan, and A. J. Shields, Experimental quantum key distribution beyond the repeaterless secret key capacity, Nat. Photonics 13, 334 (2019).

[21] M. K. Bhaskar, R. Riedinger, B. Machielse, D. S. Levonian, C. T. Nguyen, E. N. Knall, H. Park, D. Englund, M. Lončar, 
D. D. Sukachev, and M. D. Lukin, Experimental demonstration of memory-enhanced quantum communication, Nature (London) 580, 60 (2020).

[22] J. Borregaard, P. Kómár, E. M. Kessler, M. D. Lukin, and A.S. Sørensen, Long-distance entanglement distribution using individual atoms in optical cavities, Phys. Rev. A 92, 012307 (2015).

[23] M. Uphoff, M. Brekenfeld, G. Rempe, and S. Ritter, An integrated quantum repeater at telecom wavelength with single atoms in optical fiber cavities, Appl. Phys. B 122, 46 (2016).

[24] A. Reiserer and G. Rempe, Cavity-based quantum networks with single atoms and optical photons, Rev. Mod. Phys. 87, 1379 (2015).

[25] T. Wilk, S. C. Webster, A. Kuhn, and G. Rempe, Single-atom Single-photon quantum interface, Science 317, 488 (2007).

[26] S. Langenfeld, O. Morin, M. Körber, and G. Rempe, A network-ready random-access qubits memory, npj Quantum Inf. 6, 86 (2020).

[27] S. Welte, B. Hacker, S. Daiss, S. Ritter, and G. Rempe, Photon-Mediated Quantum Gate between Two Neutral Atoms in an Optical Cavity, Phys. Rev. X 8, 011018 (2018).

[28] C. H. Bennett and G. Brassard, Quantum cryptography: public key distribution and coin tossing, in Proceedings of the IEEE International Conference on Computers, Systems and Signal Processing (New York, 1984) pp. 175-179.

[29] V. Scarani, H. Bechmann-Pasquinucci, N. J. Cerf, M. Dušek, N. Lütkenhaus, and M. Peev, The security of practical quantum key distribution, Rev. Mod. Phys. 81, 1301 (2009).

[30] Y.-A. Chen, S. Chen, Z.-S. Yuan, B. Zhao, C.-S. Chuu, J. Schmiedmayer, and J.-W. Pan, Memory-built-in quantum teleportation with photonic and atomic qubits, Nat. Phys. 4, 103 (2008).

[31] M. Brekenfeld, D. Niemietz, J. D. Christesen, and G. Rempe, A quantum network node with crossed optical fibre cavities, Nat. Phys. 16, 647 (2020).

[32] See supplemental material at http://link.aps.org/supplemental/ 10.1103/PhysRevLett.126.230506 for a detailed description of the experimental sequence, dynamical decoupling, Bell-state measurement and data processing, which includes Refs. [17,26,33-35].

[33] M. Körber, O. Morin, S. Langenfeld, A. Neuzner, S. Ritter, and G. Rempe, Decoherence-protected memory for a singlephoton qubit, Nat. Photonics 12, 18 (2018).

[34] H. Y. Carr and E. M. Purcell, Effects of diffusion on free precession in nuclear magnetic resonance experiments, Phys. Rev. 94, 630 (1954).

[35] S. Meiboom and D. Gill, Modified spin-echo method for measuring nuclear relaxation times, Rev. Sci. Instrum. 29, 688 (1958).

[36] S. Muralidharan, L. Li, J. Kim, N. Lütkenhaus, M. D. Lukin, and L. Jiang, Optimal architectures for long distance quantum communication, Sci. Rep. 6, 20463 (2016).

[37] J. P. Covey, I. S. Madjarov, A. Cooper, and M. Endres, 2000-Times Repeated Imaging of Strontium Atoms in Clock-Magic Tweezer Arrays, Phys. Rev. Lett. 122, 173201 (2019).

[38] R. Trényi and N. Lütkenhaus, Beating direct transmission bounds for quantum key distribution with a multiple quantum memory station, Phys. Rev. A 101, 012325 (2020).

[39] G. Murta, F. Grasselli, H. Kampermann, and D. Bruß, Quantum conference key agreement: A review, Adv. Quantum Technol. 3, 2000025 (2020). 